\title{
TNF- $\alpha$ promotes tumor lymph angiogenesis in head and neck squamous cell carcinoma through regulation of ERK3
}

\author{
Caiyun Zhang", Minhui Zhu", Wei Wang", Donghui Chen, Shicai Chen, Hongliang Zheng \\ Department of Otorhinolaryngology-Head and Neck Surgery, Changhai Hospital, Navy Medical University, Shanghai 200433, China \\ Contributions: (I) Conception and design: H Zheng; (II) Administrative support: H Zheng; (III) Provision of study materials or patients: C Zhang; (IV) \\ Collection and assembly of data: C Zhang, D Chen; (V) Data analysis and interpretation: M Zhu, S Chen; (VI) Manuscript writing: All authors; (VII) \\ Final approval of manuscript: All authors. \\ "These authors contributed equally to this work. \\ Correspondence to: Hongliang Zheng. Department of Otorhinolaryngology-Head and Neck Surgery, Changhai Hospital, Navy Medical University, \\ No. 168 Changhai Road, Shanghai 200433, China. Email: zheng_hl2004@163.com.
}

\begin{abstract}
Background: Squamous cell carcinoma of the head and neck (HNSCC) is the sixth most common cancer worldwide. The poor prognosis of HNSCC patients is largely due to its early lymph node metastasis. Tumor necrosis factor (TNF)- $\alpha$ has been reported to be involved in lymph angiogenesis in several cancers whereas its relationship with HNSCC was still largely unknown.
\end{abstract}

Methods: In this work, mice xenograft model was constructed and treated with extra TNF- $\alpha$, Hep-2 conditioned medium was constructed for human lymphatic endothelial cells (hLECs) culture. qRT-PCR, Western blot analysis and immunohistochemical (IHC) staining were utilized to detect the expression levels of lymph angiogenesis markers as well as ERK3 and its downstream. CCK8 assay was performed to evaluate cell proliferation. Wound healing assay was used for measuring cell migration. Tube formation assay was used to estimate the tube formation ability of hLECs.

Results: The results indicated that TNF- $\alpha$ could up-regulated the expression levels of VEGF-C, VEGFR-3 and LYVE-1 in cell or animal level, change the cell morphology, accelerate cell proliferation, enhance cell migration and tube formation ability of hLECs. Moreover, ERK3 was up-regulated by TNF- $\alpha$ in both cell and animal levels and the over-expression of ERK3 in hLECs could also up-regulate VEGF-C and promote cell proliferation.

Conclusions: In conclusion, this study indicated the promotion effect of TNF- $\alpha$ on tumor lymph angiogenesis in HNSCC and demonstrated that ERK3 may be involved in the underlying molecular mechanism. This work provides new diagnostic and therapeutic targets, which are of great significance to improve the prognosis of HNSCC patients.

Keywords: Squamous cell carcinoma of the head and neck (HNSCC); TNF- $\alpha$; ERK3; lymph angiogenesis; human lymphatic endothelial cells (hLECs)

Submitted May 15, 2019. Accepted for publication Sep 12, 2019.

doi: $10.21037 /$ tcr.2019.09.60

View this article at: http://dx.doi.org/10.21037/tcr.2019.09.60

\section{Introduction}

Squamous cell carcinoma of the head and neck (HNSCC), which generally includes malignant tumor covered by squamous epithelium occurs in the oral cavity, pharynx and larynx, is the sixth most common cancer worldwide $(1,2)$. In spite of the development of resection and radiotherapy against HNSCC, the prognosis of HNSCC patients is still poor because of its early lymph node metastasis, which is one of the most annoying characteristic of cancer $(3,4)$. The development of malignant tumors commonly is accompanied by lymph angiogenesis. However, more and more evidence showed that tumor-induced lymph 
angiogenesis plays an important role in promoting tumor growth, especially lymph node metastasis (5). It has been reported that the high lymphatic vessel density is significantly correlated with cervical lymph node metastasis (6). Therefore, the mechanism of tumor lymph angiogenesis can provide important theoretical basis for lymph node metastasis, and explore new diagnostic and therapeutic targets, which is of great significance to improve the prognosis of HNSCC. Although vascular endothelial growth factor (VEGF)-C and VEGF-D have been identified as two relatively high specific lymphatic endothelial markers, the molecular mechanism of the regulation of lymph angiogenesis by HNSCC is still largely unclear (7).

As an important inflammatory cytokine, tumor necrosis factor (TNF)- $\alpha$ plays an important role in inflammation, immune stress, cell homeostasis, and incidence and development of malignant tumors $(8,9)$. Studies have shown that TNF- $\alpha$ participates in almost every stage in the occurrence and development of cancer, including malignant transformation, proliferation, angiogenesis, invasion and metastasis of tumor cells (10). Actually, in many pathological conditions, the occurrence of inflammation is closely and indivisibly related to lymph angiogenesis (11). During the research of some non-neoplastic diseases, it was revealed that TNF- $\alpha$ could promote the formation of lymphatic vessels by regulating the proliferation of lymphatic endothelial cells (12). Moreover, TNF- $\alpha$ could up-regulate the mRNA and protein level of VEGF-C, which is a wellknown promotion factor for tumor lymph angiogenesis, in cells such as fibroblasts and rheumatoid synoviocytes $(13,14)$. More importantly, it has also been reported that TNF- $\alpha$ was directly involved in the tumor lymph angiogenesis (15). However, the involvement of TNF- $\alpha$ in lymph angiogenesis of head and neck squamous cell carcinoma has not been reported. Our previous work demonstrated that TNF- $\alpha$ is significantly associated with recurrence and lymph node metastasis of oropharyngeal squamous cell carcinoma, especially in HPV16 positive patients, indicating the potential involvement of TNF- $\alpha$ in the tumor lymph angiogenesis of HNSCC $(16,17)$.

Mitogen-activated protein kinase (MAPK) is a group of serine-threonine protein kinases which could be activated by different extracellular stimuli, such as cytokines, neurotransmitters, hormones, cell stress and cell adhesion (18). This signaling pathway is involved in cell proliferation, differentiation and apoptosis, and is closely related to the occurrence and development of tumors (18). As an atypical member of MAPK family which was originally identified in 1991, extracellular signalregulated kinase (ERK)-3 was found to be up-regulated in several human tumors such as non-small cell lung cancer, gastric cancer and breast cancer and closely related to tumor invasion and metastasis (19). Although there are only few studies on the molecular regulation mechanism of ERK3, the latest research has illustrated that TNF- $\alpha$ could induce the up-regulation of ERK3 through the transcription factor c-Jun, thus up-regulating VEGFR-2 and stimulating the proliferation, migration of vascular endothelial cells and angiogenesis (20). Considering the similarity of vascular angiogenesis and lymph angiogenesis, we hypothesized that TNF- $\alpha$ could induce tumor lymph angiogenesis in HNSCC through the up-regulation of ERK3 and aimed to verify the deduction in this work.

\section{Methods}

\section{Cell lines and cell culture}

The human oral squamous cell carcinoma cell lines Hep-2 and human lymphatic endothelial cells (hLECs) were obtained from BeNa Culture Collection (Hangzhou, Zhejiang, China). hLEC were cultured with $85 \%$ RPMI1640 (Thermo Fisher Scientific, Waltham, MA, USA) and $15 \%$ FBS (Atlas Biologicals), and Hep-2 were maintained in RPMI-1640 (Thermo Fisher Scientific, Waltham, MA, USA) and $10 \% \mathrm{FBS}$ at $37^{\circ} \mathrm{C}$ in a humidified atmosphere containing $5 \% \mathrm{CO}_{2}$.

The hLEC cells were plated in a six-well plate and treated with EBM-2 or Hep-2 conditioned medium for $48 \mathrm{~h}$. Then the cells were collected and used to extract protein and RNA for correlation detection (western blot analysis and qRT-PCR).

\section{HNSCC mice xenograft model construction}

Six-week-old nude mice, weighing approximately $14 \mathrm{~g}$ were purchased from Jiesijie Company (Shanghai, China), and housed under a $12 \mathrm{~h}$ light/dark cycles at $24-25^{\circ} \mathrm{C}$ with a relative humidity of $50-55 \%$ conditions. All animal experimental protocols were approved by the Committee for the Handling and Use of Animals at Changhai Hospital, Navy Medical University.

Hep-2 $\left(4 \times 10^{6}\right)$ cells were injected directly into nude mice to construct a mice xenograft model. After tumor cell implantation, mice in TNF- $\alpha$ group were treated by TNF- $\alpha$ 
$(0.1 \mathrm{mg} / \mathrm{Kg} \cdot \mathrm{Bw})$ through intravenous (tail vein) injection and mice in control group were injected by corresponding saline. After 2-3 weeks of culture, these mice were sacrificed and the tumor were collected for subsequent experiment and the number of lymphangiogenesis inside and around the tumor were counted.

\section{Immunobistochemical staining}

Head and neck squamous cell carcinoma tissue sections from the carcinoma nude mice models were deparaffinized. After citrate antigen repairing and 20 min blocking with goat serum $(1: 20)$, the samples were incubated with anti-LYVE-1 (1:500) (Abcam, \# ab14917, Cambridge, MA, USA) at $4{ }^{\circ} \mathrm{C}$ overnight in the incubator. Tissue sections were stained with $\mathrm{DAB}$ for $10 \mathrm{~min}$, and again stained with hematoxylin. Images were captured using a photomicroscope and analyzed (Olympus, Shinjuku, Tokyo, Japan). The expression of LYVE-1 (LOD value) was quantified with ImageJ.

\section{RNA extraction and $q R T-P C R$}

After $24 \mathrm{~h}$ cultivation of hLECs, the mRNA level of ERK3, TNF- $\alpha$, VEGF-C and VEGFR- 3 was measured in the Hep-2 conditioned medium by qRT-PCR. Briefly, total mRNA was collected with trizol reagent (Invitrogen, Carlsbad, CA, USA) according to the manufacturer's instruction, and quantified by Nanodrop 2000/2000C (Thermo Scientific, Waltham, MA, USA). cDNA was obtained using the Promega M-MLV System (Promega, Madison, WI, USA) following the manufacturer's instructions. PCR forward and reverse primer sequences were designed. PCR detecting conditions were set as $95^{\circ} \mathrm{C}$ $10 \mathrm{~s}, 1$ cycle; $95^{\circ} \mathrm{C} 5 \mathrm{~s}, 60^{\circ} \mathrm{C} 30 \mathrm{~s}, 45$ cycles. The relative mRNA expression was calculated with $2^{-\Delta \Delta C T}$ method, using GAPDH as an internal control. Nanodrop 2000/2000C spectrophotometric were used to analysis the relative levels of mRNAs and the quality of extracted RNA.

\section{Western blot analysis}

In order to investigate expression level of ERK3, TNF- $\alpha$, VEGF-C and VEGFR-3, hLEC cells were collected and lysed by RIPA lysis buffer (Cell Signal Technology, Danvers, MA, USA) on ice according to the manufacturer's instruction. Then, the total cellular proteins were subjected to SDS-PAGE (10\%) for western analysis.
After transferring to polyvinylidene difluoride (PVDF) membranes, blots were incubated with 5\% BSA (Gibco, Rockville, MD, USA) in Tris buffered saline containing $0.5 \%$ Tween 20 for $60 \mathrm{~min}$. Then incubated overnight at $4{ }^{\circ} \mathrm{C}$ on a rocker with the following primary antibodies: anti-ERK3 (SAB, \#33914), anti-TNF- $\alpha$ (abcam, \#ab6671), Anti-VEGF antibody (SAB, \#41702) and VEGFR3 (SAB, \#44895) antibody (1:1,000). Following washing three times with TBST for $5 \mathrm{~min}$, membranes were incubated with horseradish peroxidase (HRP) conjugated goat anti-rabbit IgG polyclonal secondary antibody $(1: 3,000)$ (Beyotime, \#A0216, Beijing, China) at room temperature for $1 \mathrm{~h}$. Using Amersham's ECL + plusTM Western blot system kit for color developing. Signals were detected with enhanced chemiluminescence, using GAPDH as the internal standard (Kodak, Rochester, NY, USA).

\section{Wound healing assay}

The hLEC cells were plated in a 24-well plate for culture. When the cell density reached $90 \%$ the cells were replaced with the EBM-2 and Hep-2 conditioned medium for scratching test. A line wound was made by scraping $100 \mu \mathrm{L}$ tips across the confluent cell layer. After washing the cells for three times, the medium were added to remove detached and damaged cells and incubated for $72 \mathrm{~h}$; wound photographs were captured using a light microscope (DFC500; Leica, Wetzlar, Germany) at 0, 12, 24, 48 and $72 \mathrm{~h}$ and the width of the scratch wounds were analyzed.

\section{bLEC cytotubule formation assay}

Matrigel was dissolved and the 96-well plate was precooled overnight at $4{ }^{\circ} \mathrm{C}$. Matrigel was diluted with $1: 1$ serum-free and growth factors supplemented medium. After mixing, 2 conditioned media (EBM-2, Hep-2 conditioned medium) were added and $50 \mu \mathrm{L}$ per well to the 96 -well plate and incubated at $37^{\circ} \mathrm{C}$ for $30 \mathrm{~min}$. Added 10,000 to 15,000 cells per well for $48 \mathrm{~h}$ culturing in an incubator, and then the tube-like structure were observed at the point of 0,24 and $48 \mathrm{~h}$ with a fluorescence microscope.

\section{CCK8 assay}

CCK8 assay was used to detect the hLECs proliferation activity which were plated in 96-well plates with 2,000 cells per well and two culture media (EBM-2 and Hep-2 conditioned medium) were added to the culture 

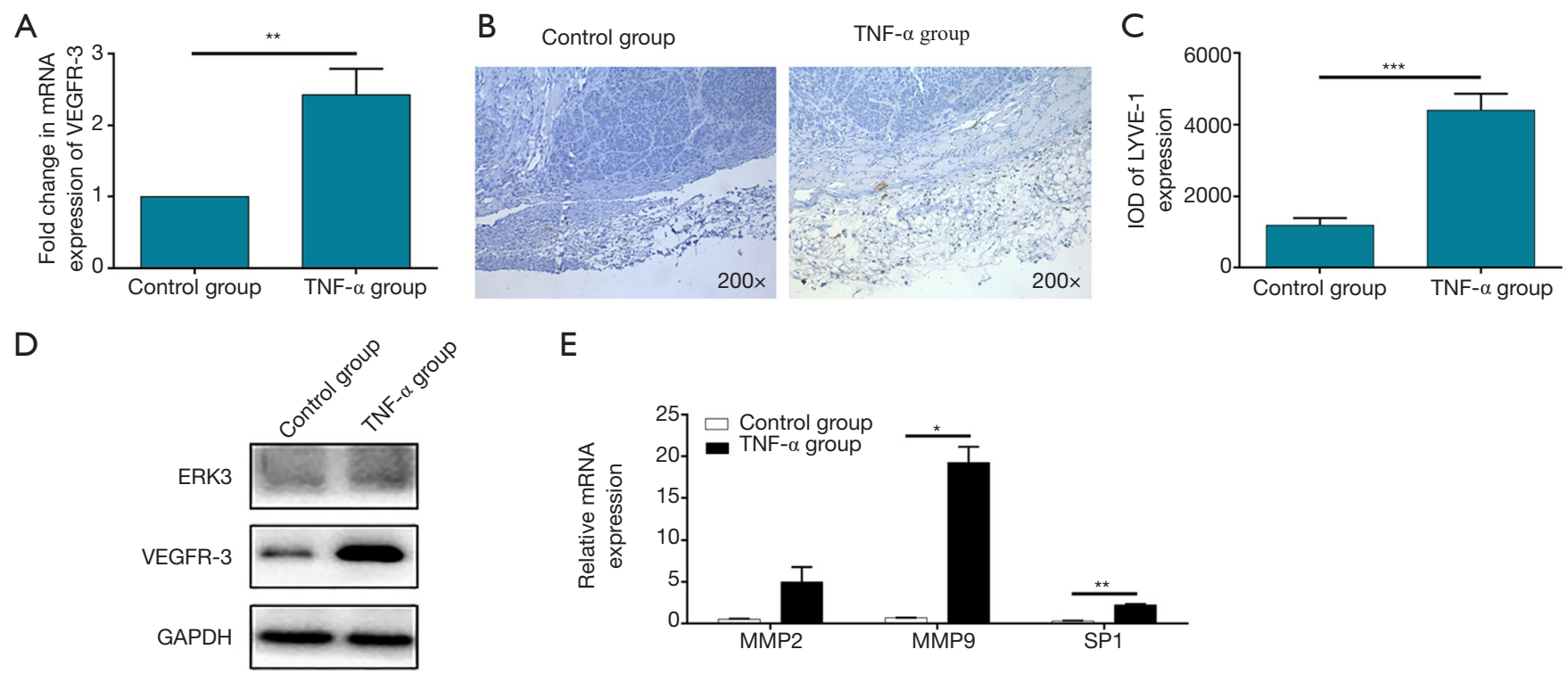

Figure 1 The effect of TNF- $\alpha$ treatment on lymph angiogenesis of HNSCC mice xenograft model. (A) Relative mRNA expression of VEGFR-3 in HNSCC mice xenograft model with (control group) or without (TNF- $\alpha$ group) TNF- $\alpha$ treatment was detected by qRT-PCR; (B,C) the expression of LYVE-1 in HNSCC mice xenograft model with (control group) or without (TNF- $\alpha$ group) TNF- $\alpha$ treatment was detected by IHC; (D) the expression of ERK3 and VEGFR-3 in control group and TNF- $\alpha$ group were detected by Western blot; (E) relative mRNA expression of MMP2, MMP9 and SP1 in control group and TNF- $\alpha$ group were detected by qRT-PCR. The data were expressed as mean $\pm \mathrm{SD}$, $\mathrm{P}$ value less than 0.05 was considered statistically significant. * $\mathrm{P}<0.05$; **, $\mathrm{P}<0.01$; *** $\mathrm{P}<0.001$. TNF, tumor necrosis factor; HNSCC, squamous cell carcinoma of the head and neck; hLECs, human lymphatic endothelial cells.

plate, respectively, and incubated four $72 \mathrm{~h}$. The assays were performed at $0,24,48$ and $72 \mathrm{~h}$ with the Cell Counting Kit-8 kit with a 96-well cell plate reader and then were examined at $450 \mathrm{~nm}$ using a microplate reader.

\section{ERK3 overexpression plasmid construction and transfection}

ERK3 overexpression plasmid were designed and constructed by Shanghai Bioscienceres, Co., Ltd. (Shanghai, China). The plasmids were transfected into hLECs using Lipofectamine 2000 transfection reagent (Thermo Fisher Scientific, Waltham, MA, USA). The validity and efficiency of ERK3 over-expression were detected and verified by qRT-PCR ( 5-fold over-expression) and Western blot analysis, respectively.

\section{Statistical analysis}

qRT-PCR was analyzed by $2^{-\Delta \Delta C T}$ method. The data are expressed as mean \pm SD and analyzed using GraphPad Prism 6 software (GraphPad Software Inc., San Diego, CA, USA).
Two-tailed $t$-test was used to compare the difference. $\mathrm{P}$ values less than 0.05 were considered statistically significant.

\section{Results}

\section{The effect of TNF- $\alpha$ treatment on lymph angiogenesis of HNSCC mice xenograft model}

In order to verify the ability of TNF- $\alpha$ to induce lymph angiogenesis in HNSCC, Hep-2 cells were utilized to construct HNSCC mice xenograft model. Subsequently, mice in TNF- $\alpha$ group were treated by TNF- $\alpha$ through intravenous injection and mice in control group were injected by corresponding saline. As shown in Figure 1A, the RT-PCR results demonstrated that the mRNA expression level of VEGFR-3, which is also a wellknown biomarker for lymph angiogenesis, was significantly up-regulated in TNF- $\alpha$ group $(\mathrm{P}<0.01)$, clearly indicating the ability of TNF- $\alpha$ to promote lymph angiogenesis. Besides, another marker for lymphatic endothelial cells lymphatic vessel endothelial hyaluronan receptor (LYVE)-1 was also proved to be distinctly up-regulated $(\mathrm{P}<0.001)$ 
A

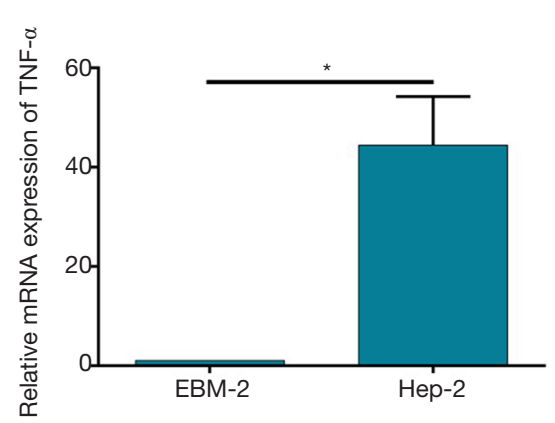

B

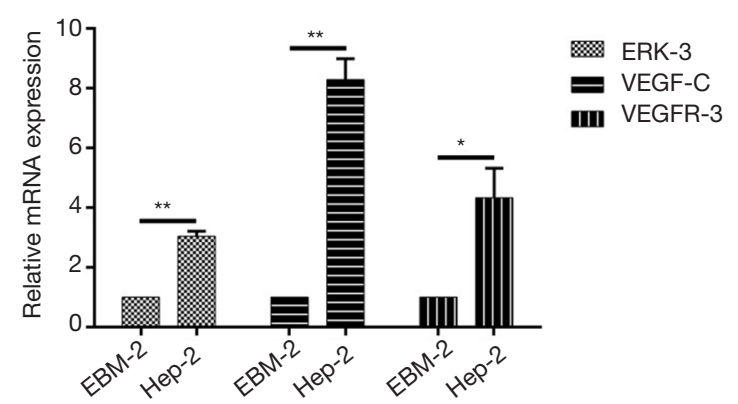

C

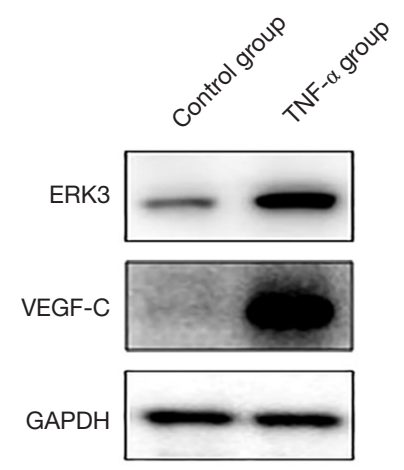

Figure 2 The effect of TNF- $\alpha$ on expression of related proteins in hLECs. (A) Relative mRNA expression of TNF- $\alpha$ in EBM-2 and Hep-2 conditioned medium; (B) relative mRNA expression of ERK3, VEGF-C and VEGFR-3 in hLECs cultured with EBM-2 or Hep-2 conditioned medium were detected by qRT-PCR; (C) the protein expression of ERK3 and VEGF-C in hLECs cultured with EBM-2 or Hep-2 conditioned medium was detected by Western blot. The data were expressed as mean $\pm \mathrm{SD}, \mathrm{P}$ value less than 0.05 was considered statistically significant. *, $\mathrm{P}<0.05$; **, $\mathrm{P}<0.01$. TNF, tumor necrosis factor; hLECs, human lymphatic endothelial cells.

which was determined by immunohistochemistry (IHC) staining (Figure 1B,C). The above results suggested that $\mathrm{TNF}-\alpha$ may possess the potential to regulate lymph angiogenesis in HNSCC.
For the sake of exploring the underlying mechanism of the promotion effect of TNF- $\alpha$, Western blot analysis was performed to detect the protein level of related signaling pathway. As shown in Figure 1D, the protein level of VEGFR-3 was significantly up-regulated by the treatment of TNF- $\alpha$, which was in consistent with the abovementioned results of RT-PCR. More importantly, it was also demonstrated that treatment of TNF- $\alpha$ induced the up-regulation of ERK3 simultaneously, indicating the potential mediating effect of ERK3. Furthermore, the up-regulation of the mRNA levels of the downstream, including matrix metalloproteinase (MMP)2, MMP9 and SP-1, of ERK3 also proved the previous results (Figure 1E).

\section{The effect of TNF- $\alpha$ on expression of related proteins in bLECs}

Given the results obtained from the animal experiments, the effect of TNF- $\alpha$ on the tumor lymph angiogenesis in HNSCC should further be studied in cell level. Herein, a conditioned medium was constructed by culturing Hep-2 cells, which is a commonly used cell line for HNSCC research, for $24 \mathrm{~h}$ and utilized to culture human lymphatic endothelial cells hLECs to simulate the microenvironment of HNSCC. After $24 \mathrm{~h}$ cultivation of hLECs, the mRNA level of TNF- $\alpha$ was measured in the Hep-2 conditioned medium by qRT-PCR, the results of which showed significant up-regulation of TNF- $\alpha$ mRNA level compared with the normal medium for hLECs (EBM-2), proving the high TNF- $\alpha$ concentration environment of the conditioned medium (Figure 2A). Subsequently, further qRT-PCR and Western blot analysis assays demonstrated that the mRNA expression levels of VEGF-C and VEGFR-3 and the protein level of VEGF-C were up-regulated, respectively (Figure 2B,C). Actually, the up-regulation of VEGFR-3 was perfectly in accordance with the results from animal models. Moreover, as the exploration of mechanism, the mRNA and protein levels of ERK3 in hLECs were also detected and both showed a similar uptrend with the animal experiments (Figure 2B,C). Therefore, the studies in cell level showed the up-regulation of both tumor lymph angiogenesis markers VEGF-C and VEGFR-3, and the up-regulation of ERK3 suggested the potential mechanism.

The effect of TNF-a on morphology, proliferation, migration and tube formation of bLECs

Next, the effect of TNF- $\alpha$ on the morphology and cellular 

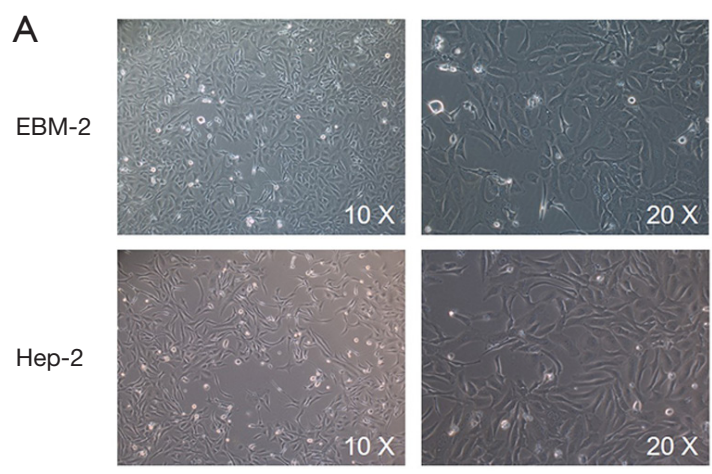

B
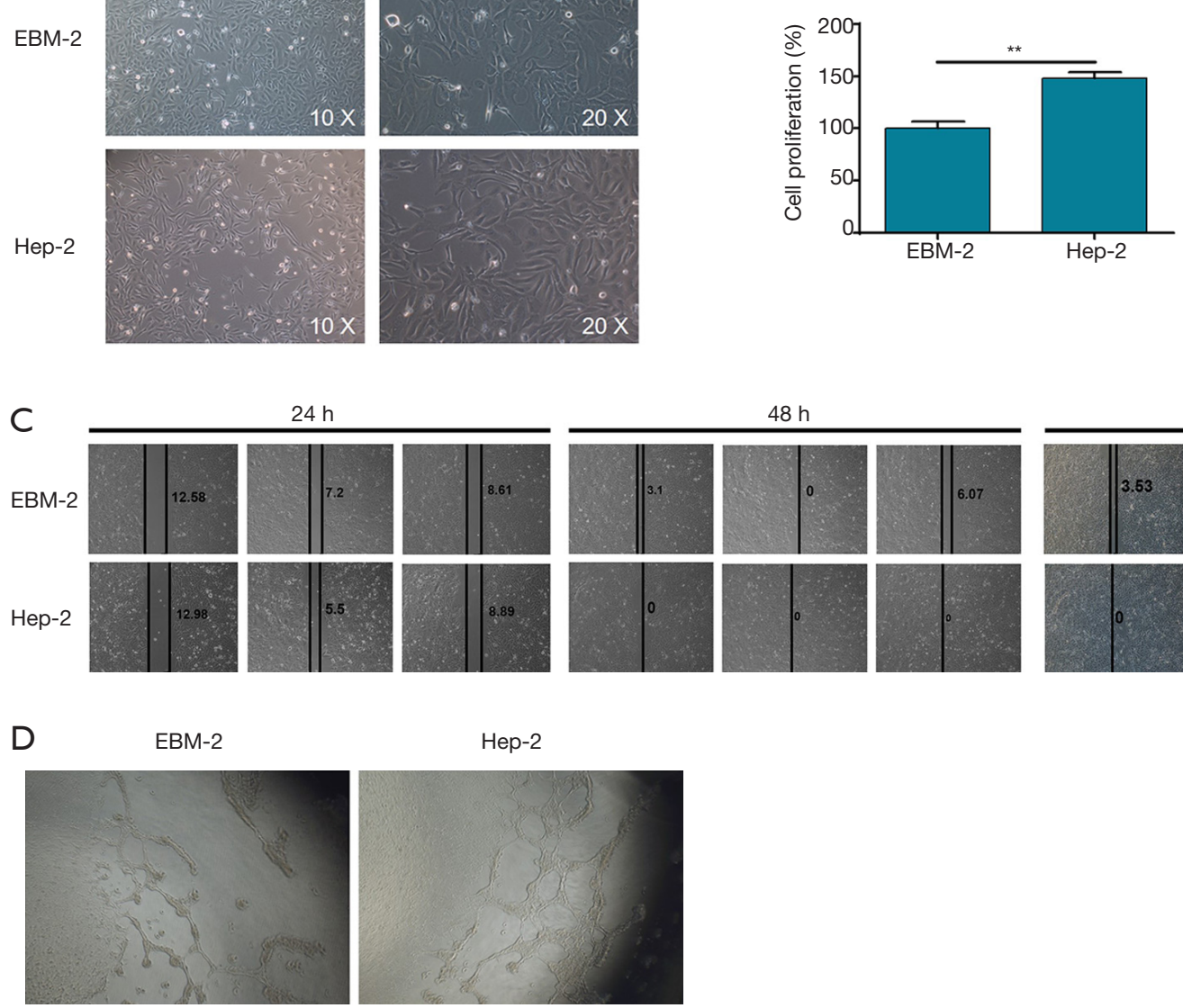

$48 \mathrm{~h}$

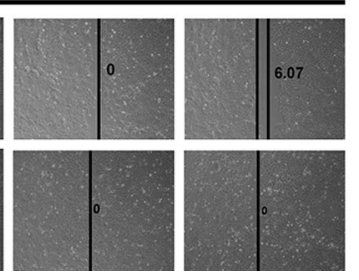

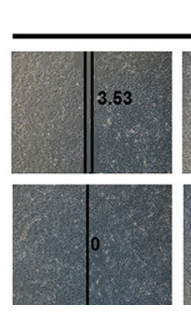

$72 \mathrm{~h}$

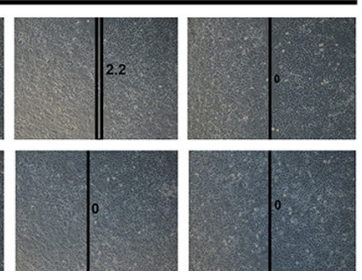

Hep-2

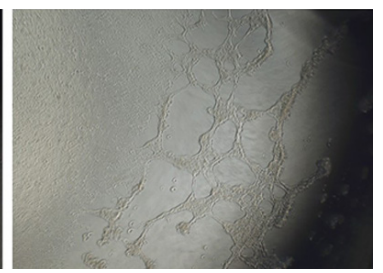

Figure 3 The effect of TNF- $\alpha$ on morphology, proliferation, migration and tube formation of hLECs. (A) The cell morphology of hLECs cultured with EBM-2 or Hep-2 conditioned medium; (B) cell proliferation of hLECs cultured with EBM-2 or Hep-2 conditioned medium; (C) the migration ability of hLECs cultured with EBM-2 or Hep-2 conditioned medium detected by wound healing assay; (D) the tube formation ability of hLECs cultured with EBM-2 or Hep-2 conditioned medium. The data were expressed as mean \pm SD, $\mathrm{P}$ value less than 0.05 was considered statistically significant. ${ }^{* *}, \mathrm{P}<0.01$. TNF, tumor necrosis factor; hLECs, human lymphatic endothelial cells.

biological behaviors of hLEC was investigated. As shown in Figure 3A, after the cultivation of hLECs in the Hep-2 conditioned medium for $48 \mathrm{~h}$, observation of cell morphology exhibited that the cells became more narrow and elongated with more pseudo convex filamentous projections, which is similar with epithelial mesenchymal transition (EMT) to some extent. Moreover, the CCK8 assay showed an apparently higher rate of cell proliferation of hLECs cultured with Hep-2 conditioned medium than the EBM-2 medium, indicating the promotion effect of TNF- $\alpha$ to cell proliferation of hLECs (Figure 3B). The wound healing assay (Figure 3C) displayed that, compared with the EBM-2 medium with relatively lower level of TNF- $\alpha$, the Hep- 2 conditioned medium with high level of TNF- $\alpha$ could increase the migration ability of hLECs. Importantly, it was also demonstrated that the tube formation ability of hLECs could be slightly enhanced by the Hep-2 conditioned medium (Figure 3D). Taken together, the above results clearly showed that TNF- $\alpha$ could change the cell morphology, promote cell proliferation, increase cell migration ability and enhanced tube formation ability of hLECs, indicating its potential to induce tumor lymph angiogenesis and lymphatic metastasis.

\section{The effect of ERK3 over-expression on hLECs}

In order to further verify the mediating role of ERK3 in the promotion effect of TNF- $\alpha$ to lymph angiogenesis in 
A

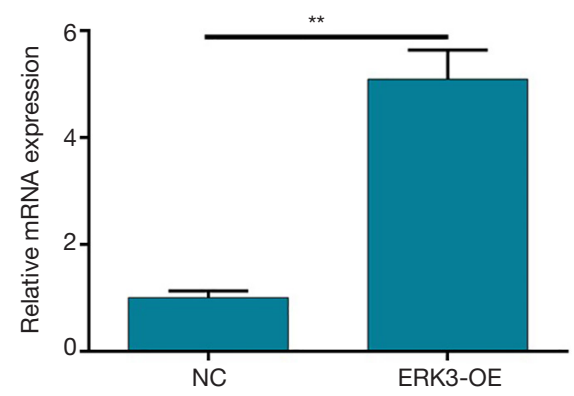

C

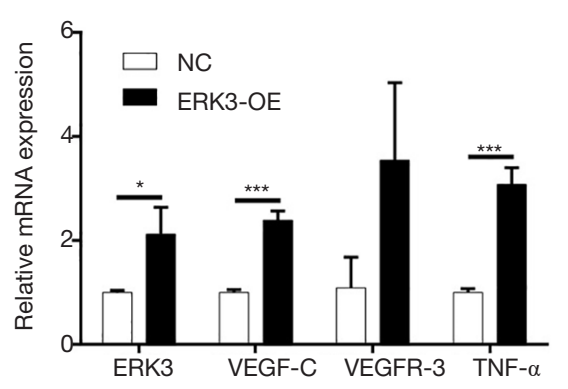

$\mathrm{E}$

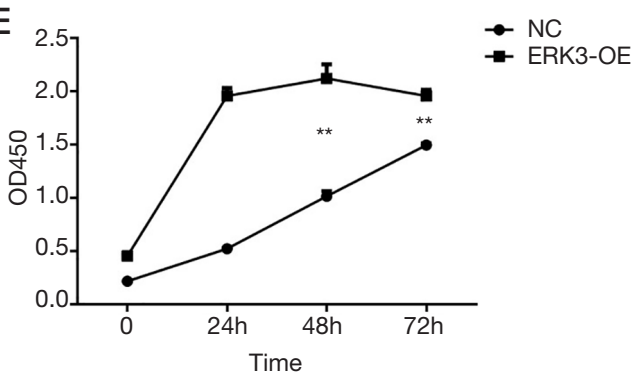

B NC ERK3-OE

ERK3

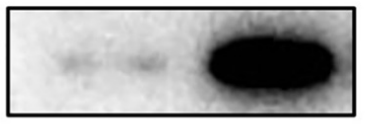

GAPDH

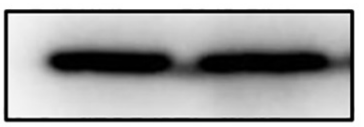

D

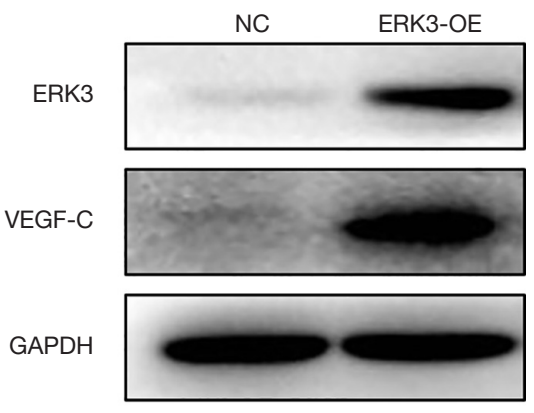

Figure 4 The effect of ERK3 over-expression on hLECs. (A,B) ERK3 over-expression was confirmed by qRT-PCR and Western blot; (C) the effects of ERK3 over-expression on relative mRNA expression of ERK3, VEGF-C, VEGFR-3 and TNF- $\alpha$; (D) the effects of ERK3 over-expression on protein expression of VEGF-C; (E) the effects of ERK3 over-expression on cell proliferation of hLECs. The data were expressed as mean $\pm \mathrm{SD}, \mathrm{P}$ value less than 0.05 was considered statistically significant. ${ }^{*}, \mathrm{P}<0.05 ;{ }^{* *}, \mathrm{P}<0.01$; ${ }^{* * *}, \mathrm{P}<0.001$. TNF, tumor necrosis factor; hLECs, human lymphatic endothelial cells.

HNSCC, the hLECs stably transfected with ERK3 overexpressed plasmid were constructed. The validity and efficiency of ERK3 over-expression were detected and verified by qRT-PCR ( 5 -fold over-expression) and Western blot analysis, respectively (Figure $4 A, B$ ). Notably, the ERK3 over-expression up-regulated the mRNA expression levels of VEGF-C and VEGFR-3 and the protein level of VEGF-C, which showed the same trend with the Hep-2 conditioned medium. Interestingly, the over-expression of ERK3 could also up-regulate the mRNA expression level of TNF- $\alpha$ (Figure $4 C, D$ ). Finally, the CCK8 assay demonstrated that, similar with the Hep-2 conditioned medium, ERK3 overexpression was also able to accelerate the cell proliferation of hLECs (Figure 4E).

\section{Discussion}

As the sixth most common malignant tumor in the world, HNSCC has poor prognosis because of its early lymph node metastasis (1). Moreover, the formation of lymphatic vessels in tumors is an important prerequisite for lymph node metastasis of malignant tumors (21). Therefore, the illustration of the mechanism of tumor lymph angiogenesis could provide important theoretical basis, and benefits for early clinical intervention, thus proving survival time and life quality of HNSCC patients. 
However, different from the hematogenous metastasis which has been intensively researched, the mechanism of lymph node metastasis is comparatively rarely reported. In spite of the questionable specificity, some molecules specifically expressed in lymphatic endothelial cells have been discovered and labeled by immunohistochemistry along with the development of molecular biology (22). At present, monoclonal antibody D2-40 and Podoplanin were the most commonly used specific biomarker for lymph angiogenesis $(23,24)$. Moreover, VEGF-C and VEGF-D are also lymphatic endothelial markers with relatively high specificity. The VEGF-C/VEGF-D/VEGFR-3 signal axis, which is mediated by them, is the major signaling pathway to promote lymph angiogenesis in a variety of solid tumors. Once VEGFR-3 is activated on the membrane of the lymphatic endothelium, VEGFR-3 tyrosine phosphorylation is induced, and a series of subsequent signaling cascade reactions lead to lymph angiogenesis, proliferation, expansion, and lymphatic metastasis of tumor cells $(7,25)$. Moreover, LYVE-1 was recently reported to be another marker for lymph angiogenesis (26). Although the specificity of LYVE-1 was limited when used alone, the combination of it with other markers such as Prox-1 and VEGFR-3 could enhance the specificity and has been successfully applied in research of sentinel lymph node formation in oral squamous cell carcinoma (27).

As the earliest and most important factor during inflammatory response, TNF- $\alpha$ plays very important role in occurrence and development of malignant tumors with both anti-cancer and cancer-promoting activity (10). More importantly, previous studies have demonstrated that TNF- $\alpha$ could induce lymph angiogenesis under specific conditions. The promotion effect of TNF- $\alpha$ on expression of VEGF-C, which is previously mentioned as lymph angiogenesis marker, and proliferation of lymphatic endothelial cells has been proved in some research of nontumor diseases $(13,14)$. It was also reported that TNF- $\alpha$ could promote the lymph angiogenesis and lymphatic metastasis of ovarian cancer through regulation of TNFR1 in a VEGFR-3-independent manner (15). Besides, TNF- $\alpha$ could also induce the lymph angiogenesis of in situ xenograft of gall bladder carcinoma in nude mice through the up-regulation of VEGF-C expression by activation of NF- $\kappa$ B signaling pathway (28). Our group has previously studied the correlation between TNF- $\alpha$ and the recurrence of HNSCC and lymph node metastasis based on clinical data. The results showed that expression of TNF- $\alpha$ was significantly and positively related with the risk of recurrence of oropharyngeal squamous cell carcinoma and lymph node metastasis, especially in HPV16 positive patients. Similarly, in patients with HNSCC in the nonoropharyngeal region, TNF- $\alpha$ is also associated with recurrence and lymph node metastasis risk $(16,17)$. These results suggested that TNF- $\alpha$ may play a role in lymph angiogenesis and lymph node metastasis in HNSCC. In this study, the role of TNF- $\alpha$ in lymph angiogenesis was further studied in animal level by using mice xenograft model constructed by Hep-2 cells with or without extra treatment of TNF- $\alpha$, and cellular level by using hLECs cultured with Hep-2 conditioned medium. The results of animal models showed that VEGFR-3 and LYVE-1, which could be used as lymph angiogenesis marker separately or in combination, could both be up-regulated by the treatment of mice models by TNF- $\alpha$. On the other hand, the cultivation of hLECs in Hep-2 conditioned medium, in which much higher TNF- $\alpha$ level was detected compared with normal EBM-2 medium, distinctly up-regulate the mRNA levels of VEGF-C and VEGFR-3 as well as the protein level of VEGF-C. Moreover, the studies concerning the cell morphology and cellular biological behaviors illustrated that the utilization of Hep-2 conditioned medium, as well as relatively high level of TNF- $\alpha$, could change cellular morphology (more narrow and elongated with more pseudo convex filamentous projections), increase cell proliferation, enhance cell migration ability and tube formation ability. Collectively, all the results elucidated that TNF- $\alpha$ played very important role in the lymph angiogenesis in HNSCC.

Among the various downstream signaling pathway that could be regulated by TNF- $\alpha$, ERKs, which are important members in MAPK family, attracted our attention because of their close relationship with tumor lymph angiogenesis (29). As an atypical member in MAPK family, ERK3 has been reported to be involved in the invasion and metastasis of various types of cancer in spite of the limited research (30). Al-Mahdi et al. indicated that the over-expression of ERK3 could induce a rounded morphology and increase the migration ability of breast cancer cells (31). Similar promotion effect of ERK 3 on the migration or invasiveness of human lung cancer cells or head and neck cancer cells was also proved in previous studies, indicating the critical role of ERK3 in invasion and migration of cancer cells (30-32). Furthermore, a recent study demonstrated that ERK3 could be up-regulated by TNF- $\alpha$ through c-Jun, which subsequently promoted endothelial cell migration, proliferation and tube formation by up-regulating SRC3/SP-1-mediated VEGFR-2 expression (20). Considering 
the similarity of angiogenesis and lymph angiogenesis, in this study, we aimed to explore whether the promotion of lymph angiogenesis by TNF- $\alpha$ in HNSCC was through the regulation of ERK3. In the research of animal models, the up-regulated expression levels of ERK3 and its downstream, including MMP2, MMP9 and SP-1 upon treatment of TNF- $\alpha$ were verified by Western blot analysis and RT-PCR, respectively. Similar uptrend of ERK3 expression was also observed in hLECs cultured with Hep-2 conditioned medium compared with EBM-2 medium. For further confirming the role of ERK3 in the promotion effect of TNF- $\alpha$ to lymph angiogenesis, hLECs with over-expressed ERK3 was constructed and verified by both RT-PCR and Western blot analysis. It was demonstrated that the over-expression of ERK3 up-regulated the mRNA levels of VEGF-C and VEGFR-3 as well as the protein level of VEGF-C, which is exactly same with the results obtained from the Hep-2 conditioned medium cultured hLECs. Moreover, CCK8 assay showed that cell proliferation of hLECs could also be accelerated by the ERK3 over-expression.

In conclusion, this study demonstrated that TNF- $\alpha$ could up-regulate the expression levels of VEGF-C, VEGFR-3 and LYVE-1, change cellular morphology, accelerate cell proliferation, enhance cell migration and tube formation ability, thus promoting tumor lymph angiogenesis in HNSCC and the regulation of ERK3 may be involved in the underlying mechanism. This work could provide new diagnostic and therapeutic targets for treatment of HNSCC and metastasis, which is of great significance to improve the prognosis of HNSCC.

\section{Acknowledgments}

Funding: This study was supported by the National Natural Science Foundation of China (Grant Number 81502361).

\section{Footnote}

Conflicts of Interest: The authors have completed the ICMJE uniform disclosure form (available at http://dx.doi. org/10.21037/tcr.2019.09.60). These authors have no conflicts of interest to declare.

Ethical Statement: The authors are accountable for all aspects of the work in ensuring that questions related to the accuracy or integrity of any part of the work are appropriately investigated and resolved. All procedures performed in studies involving human participants were in accordance with the ethical standards of the institutional and/or national research committee(s) and with the Helsinki Declaration (as revised in 2013). The institutional and written informed consent were waived. All animal experimental protocols were approved by the Committee for the Handling and Use of Animals at Changhai Hospital, Navy Medical University in compliance with the national or institutional guidelines for the care and use of animals.

Open Access Statement: This is an Open Access article distributed in accordance with the Creative Commons Attribution-NonCommercial-NoDerivs 4.0 International License (CC BY-NC-ND 4.0), which permits the noncommercial replication and distribution of the article with the strict proviso that no changes or edits are made and the original work is properly cited (including links to both the formal publication through the relevant DOI and the license). See: https://creativecommons.org/licenses/by-nc-nd/4.0/.

\section{References}

1. Torre LA, Bray F, Center MM, et al. Global cancer statistics. CA Cancer J Clin 2015;65:87-108.

2. Forastiere A, Koch W, Trotti A, et al. Head and neck cancer. New Engl J Med 1993;328:1783-4.

3. Ferlay J, Shin HR, Bray F, et al. Estimates of worldwide burden of cancer in 2008: GLOBOCAN 2008. Int J Cancer 2010;127:2893-917.

4. Siegel R, Ward E, Brawley O, et al. Cancer statistics, 2011: the impact of eliminating socioeconomic and racial disparities on premature cancer deaths. CA Cancer J Clin 2011;61:212-36.

5. Stacker SA, Williams SP, Karnezis T, et al. Lymphangiogenesis and lymphatic vessel remodelling in cancer. Nat Rev Cancer 2014;14:159-72.

6. Beasley NJ, Prevo R, Banerji S, et al. Intratumoral lymphangiogenesis and lymph node metastasis in head and neck cancer. Cancer Res. 2002;62:1315-20.

7. Sun L, Duan J, Jiang Y, et al. Metastasis-associated in colon cancer-1 upregulates vascular endothelial growth factor-C/D to promote lymphangiogenesis in human gastric cancer. Cancer Lett 2015;357:242-53.

8. Lippitz BE. Cytokine patterns in patients with cancer: a systematic review. Lancet Oncol 2013;14:e218-28.

9. Croft M, Benedict CA, Ware CF. Clinical targeting of the TNF and TNFR superfamilies. Nat Rev Drug Discov 2013;12:147-68.

10. Fischer S, Gesierich S, Griemert B, et al. Extracellular 
RNA liberates tumor necrosis factor- $\alpha$ to promote tumor cell trafficking and progression. Cancer Res 2013;73:5080-9.

11. Nihei M, Okazaki T, Ebihara S, et al. Chronic inflammation, lymphangiogenesis, and effect of an antiVEGFR therapy in a mouse model and in human patients with aspiration pneumonia. J Pathol 2015;235:632-45.

12. Bouta EM, Wood RW, Brown EB, et al. In vivo quantification of lymph viscosity and pressure in lymphatic vessels and draining lymph nodes of arthritic joints in mice. J Physiol 2014;592:1213-23.

13. Ristimäki A, Narko K, Enholm B, et al. Proinflammatory cytokines regulate expression of the lymphatic endothelial mitogen vascular endothelial growth factor-C. J Biol Chem 1998;273:8413-8.

14. Cha HS, Bae EK, Koh JH, et al. Tumor necrosis factoralpha induces vascular endothelial growth factor-C expression in rheumatoid synoviocytes. J Rheumatol 2007;34:16-9.

15. Ji H, Cao R, Yang Y, et al. TNFR1 mediates TNF- $\alpha$ induced tumour lymphangiogenesis and metastasis by modulating VEGF-C-VEGFR3 signalling. Nat Commun 2014;5:4944.

16. Zhang C, Sturgis EM, Zheng H, et al. Genetic variants in TNF- $\alpha$ promoter are predictors of recurrence in patients with squamous cell carcinoma of oropharynx after definitive radiotherapy. Int J Cancer 2014;134:1907-15.

17. Zhang C, Sturgis EM, Zheng H, et al. TNF- $\alpha$ promoter polymorphisms and risk of recurrence in patients with squamous cell carcinomas of the nonoropharynx. Int J Cancer 2014;135:1615-24.

18. Pritchard AL, Hayward NK. Molecular pathways: mitogen-activated protein kinase pathway mutations and drug resistance. Clin Cancer Res 2013;19:2301-9.

19. Boulton TG, Nye SH, Robbins DJ, et al. ERKs: A family of protein-serine/threonine kinases that are activated and tyrosine phosphorylated in response to insulin and NGF. Cell 1991;65:663-75.

20. Wang W, Bian K, Vallabhaneni S, et al. ERK3 promotes endothelial cell functions by upregulating SRC-3/ SP1-mediated VEGFR2 expression. J Cell Physiol 2014;229:1529-37.

21. Keklikoglou I, Hosaka K, Bender C, et al. MicroRNA-206 functions as a pleiotropic modulator of cell proliferation, invasion and lymphangiogenesis in pancreatic adenocarcinoma by targeting ANXA2 and KRAS genes.
Oncogene 2015;34:4867-78.

22. Cho KH, Cheong JS, Ha YS, et al. The anatomy of fetal peripheral lymphatic vessels in the head-and-neck region: an immunohistochemical study. J Anat 2012;220:102-11.

23. Kahn HJ, Marks A. A new monoclonal antibody, D2-40, for detection of lymphatic invasion in primary tumors. Lab Invest 2002;82:1255-7.

24. Parhar S, Kaur H, Vashist A, et al. Role of podoplanin in potentially malignant disorders and oral squamous cell carcinoma and its correlation with lymphangiogenesis. Indian J Cancer 2015;52:617-22.

25. Cao R, Ji H, Feng N, et al. Collaborative interplay between FGF-2 and VEGF-C promotes lymphangiogenesis and metastasis. Proc Natl Acad Sci U S A 2012;109:15894-9.

26. Gordon EJ, Gale NW, Harvey NL. Expression of the hyaluronan receptor LYVE-1 is not restricted to the lymphatic vasculature; LYVE-1 is also expressed on embryonic blood vessels. Dev Dyn 2008;237:1901-9.

27. Ishii H, Chikamatsu K, Sakakura K, et al. Primary tumor induces sentinel lymph node lymphangiogenesis in oral squamous cell carcinoma. Oral Oncol 2010;46:373-8.

28. Du Q, Jiang L, Wang X, et al. Tumor necrosis factor- $\alpha$ promotes the lymphangiogenesis of gallbladder carcinoma through nuclear factor- $\kappa \mathrm{B}$-mediated upregulation of vascular endothelial growth factor-C. Cancer Sci 2014;105:1261-71.

29. Han KY, Chang JH, Dugas-Ford J, et al. Involvement of lysosomal degradation in VEGF-C-induced downregulation of VEGFR-3. Febs Lett 2014;588:4357-63.

30. Long W, Foulds CE, Qin J, et al. ERK3 signals through SRC-3 coactivator to promote human lung cancer cell invasion. J Clin Invest 2012;122:1869-80.

31. Al-Mahdi R, Babteen N, Thillai K, et al. A novel role for atypical MAPK kinase ERK3 in regulating breast cancer cell morphology and migration. Cell Adh Migr 2015;9:483-94.

32. Elkhadragy L, Chen M, Miller K, et al. A regulatory BMI1/let-7i/ERK3 pathway controls the motility of head and neck cancer cells. Mol Oncol 2017;11:194-207.

Cite this article as: Zhang C, Zhu M, Wang W, Chen D, Chen S, Zheng H. TNF- $\alpha$ promotes tumor lymph angiogenesis in head and neck squamous cell carcinoma through regulation of ERK3. Transl Cancer Res 2019;8(6):2439-2448. doi: 10.21037/ tcr.2019.09.60 